\title{
Iniciativas sociales, económicas y ambientales: su impacto en la opinión de la base asociativa de una Cooperativa de Ahorro y Crédito de Costa Rica
}

\author{
Paul Masís Solano, Irune Gómez Pescador y \\ Paula Arzadun
}

\section{RESUMEN}

Los inicios del cooperativismo costarricense se remontan al siglo XIX, con unas primeras expresiones que perseguían la satisfacción de necesidades de productores y trabajadores agrícolas. En el año 1943, ya existiendo 23 empresas cooperativas, surgió la primera legislación a favor del sector. Actualmente existen 594 cooperativas de adultos y escolares, y 887.335 personas asociadas a ellas. De acuerdo con el último Censo Nacional Cooperativo (Instituto Nacional de Fomento Cooperativo - INFOCOOP, 2012), el 22,2\% de las cooperativas costarricenses pertenece al sector de finanzas y seguros, ocupando el primer lugar en el ranking de principal actividad económica de las cooperativas. Entre ellas se encuentra CoopeAnde $N^{\circ} 1$ R.L, foco de análisis de la presente investigación. Se trata de una entidad que surgió en el año 1965 con el propósito de brindar una solución financiera a personas del sector educación. Actualmente cuenta con 57.000 asociados en todo el país.

A partir de un trabajo de campo realizado a nivel nacional entre los meses de diciembre de 2013 y febrero de 2014, con una muestra total de 2.223 casos, se pretende medir el impacto de una serie de iniciativas sociales, ambientales y económicas en la opinión de la base asociativa, así como su percepción y valoración respecto a temáticas socioeconómicas y ambientales.

PALABRAS CLAVE: impacto, percepción, valoración, reputación, Costa Rica, cooperativismo, CoopeAnde $\mathrm{N}^{0} 1$ R.L.

CLAVES ECONLIT: P13, C23, E51.

Cómo citar este artículo / How to cite this article: MASÍS, P., GÓMEZ, I. \& ARZADUN, P. (2016): "Iniciativas sociales, económicas y ambientales: su impacto en la opinión de la base asociativa de una Cooperativa de Ahorro y Crédito de Costa Rica", CIRIEC-España, Revista de Economía Pública, Social y Cooperativa, 86, 101-122.

Correspondencia: Paul Masís Solano, doctorando en Ciencias Empresariales por la Universidad de Mondragón, MBA con énfasis en Alta Gerencia por la Universidad de San José, Costa Rica, socio fundador de AiM consultores, paul@aim.cr; Paula Arzadun, doctora en Ciencias Empresariales por la Universidad de Mondragón, profesora de la Escuela de Administración de Empresas del Tecnológico de Costa Rica, parzadun@itcr.ac.cr; Irune Gómez Pescador, doctora en Ciencias Empresariales por la Universidad de Mondragón, irugomez@gmail.com. 


\section{EXPANDED ABSTRACT}

\section{Social, economic and environmental initiatives: impact on the opinion of the membership base of a credit cooperative in Costa Rica}

The present study aims to measure the impact of a range of social, environmental and economic initiatives on the opinion of the membership base of CoopeAnde N ${ }^{\circ} 1$ R.L, as well as the members' perception and assessment of socio-economic and environmental issues. The pursuit of this general objective is based on fieldwork conducted at national level between the months of December 2013 and February 2014, with a total sample of 2223 cases. Overall, the study provides empirical evidence on the relevance of CSR actions in the perception of the membership base. Additionally, the results reflect the relevance of a strategic and cross-cutting approach to CSR in the organization.

The Costa Rican cooperative movement began early in the nineteenth century, with its first expressions pursuing the satisfaction of the needs of farm workers. In 1943, when 23 cooperative enterprises were already in existence, the first legislation for the sector was adopted. Currently there are 594 adult and school cooperatives and 887,335 people associated with them. According to the latest National Census of Cooperatives (Instituto Nacional de Fomento Cooperativo - INFOCOOP, 2012), $22.2 \%$ of Costa Rican cooperatives belong to the finance and insurance sector, which top the ranking of cooperatives by main economic activity. They include CoopeAnde N ${ }^{\circ} 1$ R.L, the focus of this research.

CoopeAnde N ${ }^{0} 1$ R.L is a cooperative that was founded in 1965 with the aim of providing a financial solution for people related to the education sector. It started business and built its capital base with 160 members. Currently, it has 57,000 members nationwide and is the leading cooperative in its sector. In 2002, CoopenAnde $\mathrm{N}^{\circ} 1$ R.L began establishing branches and decentralizing its services. Currently it has 21 branches around the country. Since the reform of the Organic Law of the Central Bank of Costa Rica (BCCR) in 1995, CoopenAnde N ${ }^{\circ}$ R.L is audited and supervised by the Superintendent of Financial Institutions (SUGEF) under the same prudential regulation scheme as for banks in Costa Rica. In addition, it is governed by the Law of Cooperative Associations, Regulatory Law for Financial Intermediation Cooperative Organizations, Organic Law of the Central Bank, regulations of the National Council of Financial System Supervision (CONASSIF) and the Labour Code, all of which provide backup and security to the members by allowing them to verify the strength and stability of the institution. 
In recent years, CoopeAnde $\mathrm{N}^{0} 1$ R.L has been seeking to adapt to a new context of increasingly complex financial markets through diverse social, economic and environmental initiatives. The general result of this process has been the presence of multiple actions targeting different stakeholders, although there is a lack of coordination among them as well as of a strategic focus. Aware of this situation, the cooperative decided to undertake an integral strategic Corporate Social Responsibility - CSR process. As part of that process, it was important to measure the impact of a range of social, environmental and economic initiatives on the opinion of its members, as well as their perception and assessment of socioeconomic and environmental issues. The results of this measurement would allow the cooperative to confirm to what extent it is promoting actions that are relevant to the needs of its membership base, as a definitive stakeholder (Mitchell et al, 1997). Such results are important for different reasons. Primarily, as stated above, so that the cooperative may identify those actions that meet the needs of its members. Additionally, because the cooperative will assign its resources and capabilities strategically to the highest priority actions. Moreover, the cooperative may identify different strategies according to the needs of the local community where its offices are located. Last but no less important than the above is so that the cooperative may know its members' perception of its CSR trajectory.

The different scenarios in which economic and financial evolution is taking place are highly complex. In consequence, multiple stakeholders generate a variety of interrelationships with each other, which, in turn, affect the enterprise-stakeholders-CSR links. Note also both that the scope and importance of CSR itself have gradually evolved and that CSR has particular peculiarities in the cooperative sector. The importance of CoopeAnde N ${ }^{\circ} 1$ R.L in the local cooperative movement and its presence around the country constitute important practical motives for this research. Equally important, several major gaps in the scientific literature related to the socioeconomic impact of cooperatives have come to light. Given the practical interest aroused by CoopeAnde N ${ }^{\circ} 1$ R.L as well as the weaknesses in the literature, this paper addresses the following main research objective: to measure the impact of a range of social, environmental and economic initiatives on the opinion of the membership base, as well as the members' perception and assessment of socio-economic and environmental issues.

The main objective was pursued on the basis of fieldwork conducted at national level between the months of December 2013 and February 2014, with a total sample of 2223 cases. The population is the membership base. It was segmented into mutually exclusive sub-groups, according to stratified sampling, based on 11 branches. The data collection tool was a semi-structured questionnaire with 17 open and closed questions. In order to lay the foundations for the analysis, the paper examines the following: specific elements of the local cooperative movement and overview of the financial sector, as well as Corporate Social Responsibility, its definitions for conventional firms and cooperative firms, and its relationship to business strategy. 
The paper concludes by providing empirical evidence on the relevance of CSR actions in the perception of the membership base. Additionally, the results reflect the importance of a strategic and crosscutting approach to CSR in the organization. In other words, multiple actions have been taken in each branch, focusing on different stakeholders. However, particularly in the social dimension, 5 out of 10 members claimed not to know the social projects of the cooperative. This was despite having found that the attributes of this dimension are those they most value.

The paper fulfils its main objective, but also identifies certain limitations, in particular related to sample size for the statistical analysis of demographic variables. These limitations and other questions that emerged during the study combine to suggest interesting directions for future research.

KEY WORDS: Impact, perception, evaluation, reputation, Costa Rica, cooperatives, CoopeAnde NN1 R.L. 


\section{1.- Introducción}

Costa Rica es un Estado democrático situado en América Central, con una extensión de 51.100 kilómetros cuadrados y una población de $4.832 .234^{1}$ de habitantes. Es de los países con mejores indicadores sociales y económicos de la región. Sin pretender ahondar en la cuestión, puede señalarse que es el país centroamericano con menor coeficiente de Gini y por ende, en el que se presenta una menor desigualdad en la distribución del ingreso. ${ }^{2}$ Cuenta con un sistema político estable, perspectivas favorables de crecimiento económico a largo plazo, desarrollo social relativamente alto, crecimiento sostenido de exportaciones no tradicionales y personas con relativa elevada cualificación (Consejo Monetario Centroamericano, 2015). Sin embargo, actualmente dos de los temas de mayor preocupación son el desempleo y la desaceleración económica (Banco Central de Costa Rica - BCCR, 2015). Por un lado, la tasa de desempleo ampliado es de $11,8 \%{ }^{3}$, y por el otro, la desaceleración se atribuye al menor ritmo de crecimiento de la industria y a la caída de la actividad agrícola.

Los últimos años han constituido un reto significativo para todos los países alrededor del orbe, pero en especial para las economías pequeñas y abiertas como la costarricense. El proceso de desaceleración en la actividad económica hace que en la actualidad las miradas se vuelquen hacia los principios de la economía social como traccionadores de la equidad y el desarrollo socioeconómico regional, capaz de disminuir la marginalidad, la extrema pobreza y las brechas sociales.

Según el IV Censo Nacional Cooperativo (Instituto de Fomento Cooperativo - INFOCOOP, 2012) el país cuenta con 376 cooperativas $^{4}$, reflejando un crecimiento de más del $12 \%$ desde comienzos del siglo XXI. De acuerdo con su principal actividad económica, "Finanzas y Seguros" se ubica en primer lugar. Entre ellas se encuentra CoopeAnde N¹ R.L, foco de análisis de la presente investigación. Se trata de una entidad que surgió en el año 1965 con el propósito de brindar una solución financiera a personas del sector educación. Actualmente cuenta con cerca de 57.000 asociados en todo el país.

A partir de un trabajo de campo realizado a nivel nacional entre los meses de diciembre de 2013 y febrero de 2014, con una muestra total de 2.223 casos, se pretende medir el impacto de una serie de iniciativas sociales, ambientales y económicas en la opinión de la base asociativa, así como su percepción y valoración respecto a temáticas socioeconómicas y ambientales.

1.- De acuerdo con el INEC-Instituto Nacional de Estadísticas y Censos de Costa Rica, http://www.inec.go.cr/Web/Home/GeneradorPagina.aspx, consultado el 26 de junio de 2015.

2.- Acorde con Databank del Banco Mundial - BM, http://datos.bancomundial.org/, consultado el 26 de junio de 2015.

3.- INEC, comparación interanual http://www.inec.go.cr/Web/Home/GeneradorPagina.aspx, consultado el 26 de junio de 2015.

4.- Cooperativas de adultos. Las estadísticas segmentan las cooperativas de adultos y escolares. Éstas últimas están establecidas en 218 centros educativos, de los cuales 149 son escuelas (68,35\%), 53 son colegios técnicos $(24,31 \%)$ y 16 son colegios académicos (7,34\%). 


\section{2.- El cooperativismo costarricense}

\subsection{Recorrido histórico}

La institución del cooperativismo en Costa Rica es considerada como la más desarrollada de América Central y la principal dentro de la Economía Social nacional. Como se mencionó anteriormente, la ideología cooperativista se torna fundamental de cara a los nuevos tiempos que se avecinan y para sobrepasar una época donde las individualidades priman sobre las colectividades y las brechas sociales se hacen cada vez más distantes; en un mundo donde la riqueza se presenta con abundancia pero muy poco equitativa en su distribución.

En Costa Rica existen referencias documentales donde se evidencia que el cooperativismo fue partícipe de los procesos sociales conformadores de una sociedad democrática, y del establecimiento de un Estado Social o Benefactor (Huaylupo, 2003a). Con respecto a este particular, fueron precisamente las sociedades de ayuda mutua precursoras del cooperativismo en Costa Rica, las que participaron activamente en los procesos que derivaron en un movimiento social que germinó la Guerra Civil de $1948^{5}$ (Huaylupo, 2003b). Estos orígenes y la participación social de los gestores del cooperativismo, así como su intervención en el movimiento revolucionario, han provocado que el cooperativismo tuviese y continúe experimentando una gran aceptación y legitimidad en la sociedad costarricense.

El hecho que no se cuente con un personaje principal en la historia inicial del cooperativismo en Costa Rica es el resultado de que este ha sido un movimiento encarnado en la sociedad, donde no es un individuo el protagonista, sino una colectividad. A pesar de las circunstancias, el movimiento cooperativista costarricense no se planteó como una forma de organización social orientada a producir una transformación violenta en la estructura de la sociedad; sino que es un sistema económico-social evolutivo y adaptativo, responsable de haber producido cambios sustentables y perdurables en el tiempo.

Sobre los hechos más formales que relatan la historia del cooperativismo costarricense, se puede indicar que los primeros intentos de agrupación cooperativista se remontan a inicios del siglo XX, cuando alrededor de 1907, un grupo de artesanos motivados por la necesidad de comercializar productos de

5.- Esta revolución a la postre fue la que propició que el 8 de mayo de 1948 se estableciera la Junta Fundadora de la Segunda República. Esta nueva organización del país emitió la Constitución Política del año 1949 y en medio de un gran debate nacional, se inició la transformación de la sociedad costarricense hacia el fortalecimiento de la democracia, alcanzando conquistas a nivel social como las Garantías Sociales y la modernización del país a nivel económico. Indudablemente, el logro más destacable de la Junta Fundadora en cuanto a la constitución de la base de la Costa Rica actual, fue haber disuelto el ejército ganador ese mismo año (1949). La abolición del ejército es una condición que aún se mantiene en Costa Rica, y la ha convertido en ejemplo, al ser el primer país en establecer esta prohibición como institución permanente, $y$ consagrarla en la Constitución Política (artículo XII del título I). 
consumo básico, se unieron con el afán de satisfacer sus necesidades y obtener más poder de negociación ante los intermediarios y comerciantes. Se denominó originariamente "Sociedad Obrera Cooperativa". El cooperativismo también estuvo representado en la principal actividad agrícola y social del agro costarricense de la época: la producción y comercialización de café. En el año de 1918 se instaura la Sociedad Cooperativa Cafetalera, integrada por pequeños productores de café de la provincia de Heredia, que eran explotados por los grandes productores, beneficiadores y exportadores de café.

Continuando con el recorrido cronológico de los hechos, es trascendente destacar que hacia finales del siglo XIX se experimentaron algunos indicios del fenómeno cooperativista costarricense inspirados en los movimientos europeos que no le eran ajenos a esta sociedad. Surgieron de este talante, una conformación de organizaciones que brindaban auxilio mutuo con préstamos a sus asociados y que constituían a su vez una fiel representación de esta época. El requerimiento de liquidez para la supervivencia de las clases más desposeídas, es la manifestación de una sociedad con una incipiente actividad mercantil, pero también es el surgimiento de nuevas desigualdades en la colectividad de Costa Rica. Estas organizaciones eran promovidas desde el exterior, no sólo por la experiencia de alemanes radicados en el país, sino también por una directa participación de españoles en la creación de cajas de ahorro (Huaylupo, 2003a).

El rol del cooperativismo costarricense y el dinámico protagonismo como eje y constructor de la sociedad, han contribuido a que este movimiento se asentase vigorosamente en el territorio y lo haya convertido en el cooperativismo más desarrollado de Centroamérica. Uno de los factores que estuvo ausente en los orígenes, como lo fue la falta de legislación, se convierte a partir de 1943 en la punta de lanza que catapulta al cooperativismo a niveles muy considerables de desarrollo y expansión6. En 1968 ya el sector cooperativista cuenta con su propia ley (No. 4179) a la cual se le hicieron múltiples reformas con el paso del tiempo. En el año de 1982 se promulga la Ley de Asociaciones Cooperativas, que recopila una serie de privilegios instituidos en aras de garantizar y promover los fines del cooperativismo y que éste desempeñe de la mejor manera su rol dentro de la sociedad.

\subsection{Las cooperativas de ahorro y crédito}

La primera cooperativa de ahorro y crédito data del año 1955. Se denominó Cooperativa Amistad o Coopeamistad R.L. y fue fundada por trabajadores de la Cooperativa de Productores de Leche COOPROLE. En la actualidad, las cooperativas de ahorro y crédito comprenden la tipología con mayor representación en Costa Rica: reúnen al $22.2 \%$, seguidas por el comercio que encarna al $12.7 \%$, y las industrias manufactureras, 12.2\% (Instituto Nacional de Fomento Cooperativo - INFOCOOP, 2012).

6.- A merced de la promulgación del código de trabajo nace, entre otras cooperativas, COOPEVICTORIA R.L., organización ubicada en Grecia de Alajuela y que es hoy la cooperativa activa más antigua de Costa Rica. Durante 1947 surge COOPROLE R.L., conocida como Dos Pinos, la cooperativa de producción que lidera el mercado de productos lácteos de Costa Rica. Ese mismo año se estableció en el Banco Nacional de Costa Rica una sección para fomentar el cooperativismo, lo que le confiere un importante impulso. Nace así, la Ley No 861 "Fomento de Cooperativas Agrícolas e Industriales". 
En el año 1984 se encargó la supervisión financiera de las cooperativas de ahorro y crédito a la Superintendencia General de Entidades Financieras - SUGEF. Este organismo regulador supervisa tanto a entidades cooperativas como no cooperativas. El hecho de que la normativa no sea diferenciada para el sector cooperativo provoca opiniones encontradas. Si bien se enfatiza la necesidad de que dichas cooperativas sean supervisadas eficientemente para darle solidez y confianza al sector, también se reconoce que se requiere cooperación entre supervisor y supervisada así como el reconocimiento de la naturaleza jurídica, las diferentes estructuras de gobierno, los diferentes tamaños y grados de complejidad de las cooperativas (Arzbach y Durán, 2010; 2011).

A inicios de la década del noventa del siglo pasado, las autoridades costarricenses crearon una ley específica para el sector de Ahorro y Crédito: la Ley de Regulación de la Actividad de Intermediación Financiera de las Organizaciones Cooperativas?.

Sin embargo, tras décadas de desarrollo del sector, en el año de 1998 ocurrió uno de los hechos más ignominiosos dentro del cooperativismo costarricense en general y del sector del ahorro y crédito en particular, generando escepticismos en torno a la transparencia y gestión ética de esta forma de organización. Ello, por la intervención del Banco Federado y BANCOOP R.L., como así también por la crisis de VIVIENDACOOP R.L. ${ }^{8}$ y COOVIVIENDA R.L. ${ }^{9}$ Estos hechos se presentaron por el negligente accionar de sus dirigentes políticos y administrativos.

Según indica la Ley de Regulación de Intermediación Financiera de Organizaciones Cooperativas, las organizaciones cooperativas de ahorro y crédito son entidades de carácter privado, de naturaleza cooperativa, que se constituyen con el propósito de promover el ahorro entre sus asociados y de crear, con el producto de esos recursos, una fuente de crédito que se les traslada a un costo razonable, para solventar sus necesidades. Así mismo, para brindarles otros servicios financieros que funcionan mediante un esquema empresarial, que les permite administrar su propio dinero sobre la base de principios democráticos y mejorar sus condiciones sociales, económicas y culturales. Según reza la misma ley, "es de interés social, la constitución y funcionamiento de estas organizaciones, como uno de los medios más eficaces para el desarrollo socioeconómico de los habitantes"10. La crisis provocada por las entidades antes mencionadas, entre los años 1998 y 1999, hizo mella en la imagen y credibilidad del sector, como también se anticipó previamente. Ese actuar se contrapuso con los fines que precisa la ley, y con las expectativas de la sociedad. Con el correr de los años, se ha observado cierta recuperación; el número

7.- Ley $n^{\circ} 7391$.

8.- El Consorcio Cooperativo de Vivienda nace como producto de una necesidad y oportunidad que las Cooperativas de Base tenían de participar en una actividad tan importante a nivel nacional como lo era el Financiamiento de Vivienda. Necesidad porque las Cooperativas enfrentaban una demanda importante en este rubro y oportunidad porque al lograr el aval del estado se tendría acceso a los recursos idóneos para atender esta actividad desde el punto de vista de precio.

9.- COOVIVIENDA nació a fines de la década del ochenta, como una empresa especializada en la intermediación financiera para la vivienda, ante el incremento de acciones dirigidas para el financiamiento de viviendas populares. Esta organización compartía el mercado con otras empresas cooperativas como UNACOOP y VIVIENDACOOP. COOVIVIENDA como órgano de segundo rango, intermediaba recursos y los colocaba en distintos agentes financieros para que a su vez sean colocados a personas. La solicitud de préstamos a COOVIVIENDA eran conseguidos a través del Banco Hipotecario para la Vivienda o captando recursos en el mercado de valores.

10.- Artículo 6, Ley $n^{\circ} 7391$ de Regulación de Intermediación Financiera de Organizaciones Cooperativas. 
de entidades cooperativas que se dedican a la actividad de ahorro y crédito ha vuelto a crecer tal como se detalla en la siguiente figura de acuerdo a los últimos tres Censos cooperativos.

\section{Gráfico 1. Evolución de la actividad financiera de las cooperativas, periodo 2000-2012}

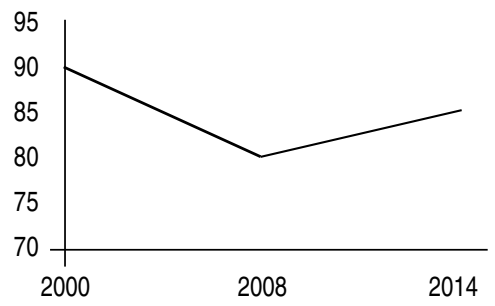

FUENTE: Elaboración propia a partir de INFOCOOP (2000, 2008, 2012).

Las cooperativas de ahorro y crédito han registrado crecimientos importantes en sus utilidades en los últimos años. Su negocio principal es el préstamo para consumo, vivienda y pequeño productor. Cuentan con convenios institucionales que les permite colocar el crédito y realizar el cobro de las cuotas por medio automático de deducción de planillas de los asociados. Asimismo, puede identificarse como denominador común una estrategia que consiste en incrementar la base asociativa y mantener cercanía con los clientes existentes.

En la actualidad, el cooperativismo costarricense recibe el reto de continuar demostrando su gran aporte histórico al desarrollo del país y el potencial como motor de la economía y el progreso nacional. Además, las cooperativas de ahorro y crédito se han de apartar del mito de que son reproductoras de pobreza. Es imperante luchar por poder contar con dirigentes más participativos. Costa Rica requiere más de cooperativistas que de administradores. Líderes que se interesen honestamente por todos los asociados, por sus necesidades y que, junto con procesos concientizados, formativos y educacionales dentro del ámbito de la organización empresarial, se propongan impulsar a la ideología cooperativa (Masís, 2009).

Si no se replantea un cambio radical, el futuro de las cooperativas de crédito será incierto. Hoy se hace necesario poder disipar las dudas y estereotipos que puedan recaer sobre esta forma de organización, para convertirla en un sistema idóneo de desarrollo socioeconómico. Desde sus inicios, han cumplido una función determinante como medio de acceso al crédito de los sectores y personas excluidas o para entes que no son sujetos de crédito por parte del sistema bancario nacional. En otras palabras, ha contribuido, y continúa haciéndolo, a la democracia socioeconómica (Organización Internacional del Trabajo - OIT, 2012). Así, ha de hacer frente a los desafíos actuales para continuar siendo competitivas y sostenibles con el propósito de cumplir con su interés social y con el desarrollo socioeconómico de la sociedad. 


\section{3.- CoopeAnde No1 R.L}

CoopeAnde №1 R.L se inició como una idea de varios integrantes de la Filial de la Asociación Nacional de Educadores (Ande) del circuito tercero de San José a inicios del año 1965, con el propósito de brindar una solución financiera a colegas del sector educación. Sin embargo, no fue hasta el 4 de Noviembre de 1965 que se celebró la Asamblea Constitutiva en la Escuela Napoleón Quesada ubicada en Zapote, donde inició su actividad y forjó su base de capital social con la integración de 160 asociados quienes aportaban $\varnothing 10$ (diez colones) ${ }^{11}$ cada uno, además, en esa ocasión se discutió y aprobó el primer estatuto de la Cooperativa y se definieron los cuerpos directores que tendrían la representación de la misma. Tuvo como primeras funciones la custodia del capital social y el de otorgar crédito a los asociados.

Durante los últimos años se ha preocupado por modernizar e innovar sus servicios y por brindar servicios eficientes y efectivos mediante el uso y aplicación de moderna tecnología en informática y telecomunicaciones, dada la amplia y constante competitividad en el Sector Cooperativo Financiero y las exigencias de Supervisión existentes en el país; consolidándose CoopeAnde №1 R.L como una Cooperativa de desarrollo que tiene trascendencia positiva en la vida económica, social y financiera de los asociados y las familias del Sector Educación de Costa Rica.

Actualmente cuenta con cerca de 57.000 asociados y asociadas entre activos y pensionados de todo el país, tanto de instituciones públicas, privadas, semi-privadas y universidades privadas y estatales de todo el territorio nacional.

Como parte de su evolución, en el año 2002 CoopeAnde №1 R.L descentralizó sus servicios con la creación de las primeras sucursales por lo que actualmente cuenta con 21 agencias ubicadas en San José, Alajuela, Heredia, Cartago, Liberia, Nicoya, Puntarenas, Pérez Zeledón, Ciudad Neily, Limón, Guápiles, San Carlos, Plaza Rofas, Desamparados, San Ramón, Guadalupe, Escazú, Cañas, Turrialba, Santa Cruz y Quepos. 


\section{4.- Responsabilidad Social Empresarial}

Como se indicó en la introducción, durante los meses de diciembre de 2013 y febrero de 2014 se llevó a cabo un trabajo de campo realizado a nivel nacional, encuestando a 2223 asociados de CoopeAnde con el propósito de conocer su opinión acerca de una serie de iniciativas sociales, ambientales y económicas así como su percepción y valoración respecto a temáticas socioeconómicas y ambientales. En otras palabras, la encuesta se basó en aspectos vinculados con la Responsabilidad Social Empresarial, cuestión que será abordada en este cuarto apartado.

Existen numerosas definiciones que tratan de esclarecer lo que se entiende por Responsabilidad Social Empresarial - RSE. Si bien no existe unanimidad de criterio, se pueden apreciar ciertas similitudes en sus interpretaciones y alcances (AECA, 2003; Comisión Europea, 2002; ISO, 2010; MTAS, 2005) vinculados con:

- la importancia de considerar y satisfacer las necesidades y demandas de los distintos grupos de interés - $\mathrm{Gl}^{12}$ que se interrelacionan con la empresa;

- la necesidad de integrar este tipo de prácticas en la planificación estratégica y en la gestión empresarial cotidiana;

- la voluntariedad de las empresas para el desarrollo de este tipo de prácticas;

- la superación del cumplimiento de la normativa legal establecida;

- la consideración de las tres dimensiones (social, ambiental y económica) en la gestión empresarial;

- la clasificación de las acciones emprendidas en función del ámbito en el que se ejercen en: internas (aquellas desarrolladas al interior de la empresa, por ejemplo medidas de seguridad laboral) y externas (las que se despliegan entre terceros agentes, por ejemplo auditorías sociales a proveedores) (Arnaez et al, 2012).

A pesar de que la RSE "va encontrando defensores y promotores dentro de las empresas grandes y medianas, pero de marcado carácter capitalista, su protagonismo se vuelve indiscutible en las empresas sociales y participativas, en las que la búsqueda del beneficio para el capital invertido no es el objetivo fundamental de su existencia" (Mugarra, 2003, p.144). Desde la primera experiencia cooperativa de Rochdale se "batalló por ser socialmente responsable en todas sus actividades" (Izquierdo Albert, 2007, p. 49).

12.- "La primera aparición del término se remonta al año 1963, en un documento interno del Stanford Research Institute. Entonces se consideraba como grupos de interés sólo a los agentes que generaban impactos sobre la empresa. Actualmente también se incluyen a todos aquellos que son afectados por la propia actividad de la organización" (Arzadun, 2011, p. 30). 
Dadas estas características, puede señalarse que las cooperativas se involucran en un proceso de ciudadanía activa, promoviendo la generación de empleo decente y mejores condiciones de vida, de forma socialmente responsable. Por ello desempeñan un importante rol en el desarrollo local y siempre han sido consideradas promotoras de transformación social. En este sentido, resulta innegable el paralelismo existente entre los principios y valores cooperativos y la filosofía promovida por la RSE (Arzadun, 2011).

El movimiento cooperativo siempre ha reconocido la importancia de satisfacer necesidades y distribuir los beneficios de su accionar de manera solidaria entre el colectivo que lo integra. Tal postura no es sólo muestra de solidaridad, sino que responde a la primacía de los intereses del conjunto sobre los fines individuales y a la búsqueda de la continuidad de la empresa. Dicha continuidad implica que la empresa cooperativa sea exitosa como empresa económica en su afán de no fracasar en su pretendida proyección social y humana (Aranzadi, 1999).

De esta manera, "visiblemente las cooperativas se insertan en una forma específica de hacer empresa, experimentando su responsabilidad social desde sus dos componentes constitutivos básicos: sus valores y principios, y su configuración empresarial. Es decir, se trata de un modelo de empresa que en lugar de centrarse en la retribución al capital económico se centra en las personas, mientras guía sus operaciones sobre la base de los valores y principios cooperativos" (Arzadun, 2012, p.150). En cuanto a su figura empresarial, la cooperativa ha de tomar en cuenta en su organización a cada grupo de interés: socios, clientes, proveedores, instituciones sociales, y demás actores que de alguna manera inciden en su obrar o sobre las cuales su funcionamiento tiene efectos o repercusiones (Belhouri et al, 2005). En este sentido, "si las experiencias y conocimientos cosechados son compartidos y transferidos, se favorece la solidaria distribución de la riqueza generada, al igual que el apoyo al desarrollo personal y profesional de cada integrante de la cooperativa. Esto termina por redundar específicamente en el progreso propio de la entidad, y finalmente de la sociedad en general (la llamada espiral de evolución) ubicando a la persona, y no al capital, en el centro de las actividades desarrolladas por la cooperativa" (Arzadun, 2012, p.151). Es por eso que se reconoce el papel de las cooperativas como agentes de desarrollo, en la medida en que colabora en la tarea de organizar los agentes económicos para generar crecimiento y desarrollo. 


\section{5.- Impacto de iniciativas sociales, económicas y ambientales en la opinión de la base asociativa de CoopeAnde N¹ R.L: datos empíricos}

En el presente apartado se detallará la ficha técnica del trabajo de campo. Los resultados se encuentran en el anexo.

\subsection{Ficha técnica}

Se ha realizado un trabajo de campo que responde a las siguientes características:

- Sitio de investigación: Costa Rica

- Universo: Asociados de CoopeAnde N¹ R.L

- Instrumento de recolección de datos: cuestionario semi-estructurado con 17 preguntas abiertas y cerradas.

- Fecha de campo: diciembre de 2013 a febrero de 2014.

- Muestreo: por cuotas13.

- Muestra: 2223 casos totales, con la distribución que se detalla seguidamente.

\section{Tabla 1. Muestra, por agencia}

\begin{tabular}{|lc|}
\hline Agencia & Base \\
\hline Cartago & 204 \\
Guápiles & 200 \\
Heredia & 205 \\
Liberia & 200 \\
Limón & 205 \\
Neily & 205 \\
Nicoya & 200 \\
Pérez Zeledón & 200 \\
Puntarenas & 204 \\
San Carlos & 200 \\
San Jose & 200 \\
Total & $\mathbf{2 2 2 3}$ \\
\hline
\end{tabular}

13.- El muestreo por cuotas comienza seleccionando unas características de control. En este caso, fue la selección de agencias. Las variables de control se escogen porque están relacionados con la característica a investigar y porque existe información sobre su distribución. Se emplean, pues, dichas variables de control para estratificar la población. A diferencia del muestreo probabilístico, se concreta el perfil de encuestado. En este estudio, correspondía que se tratara de asociados de la cooperativa (Alaminos Chica y Castehón Costa, 2006). 
- Error muestral: 2,1\% al 95\% de confianza.

- Procesamiento de datos: software estadístico SPSS.

- Escala: Escala tipo Likert de 7 puntos, siendo 1 "totalmente en desacuerdo" y 7 "totalmente de acuerdo".

- Escalas de medida

\begin{tabular}{|l|c|l|}
\hline VARIABLE & No ÍTEMS & REFERENCIA \\
\hline RSE & 27 & Alvarado (2008), Prado et al (2004) \\
CONGRUENCIA & 4 & Pérez (2011); Lafferty et al. (2002); Bigné et al. (2009) \\
MOTIVACIÓN & 4 & Pérez (2011); Dean (2002); Becker-Olsen et al. (2006); Currás (2007) \\
CREDIBILIDAD & 4 & Pérez et al. (2009); Newell y Goldsmith (2001) \\
REPUTACIÓN & 4 & Ponzi et al. (2011) \\
CALIDAD DEL SERVICIO PERCIBIDO & 12 & García de los Salmones et al., 2010 adaptación de la escala SERVQUAL (1988) \\
SATISFACCIÓN Y LEALTAD & 11 & Pérez (2011); Oliver (1980 y 1997) \\
\hline
\end{tabular}

\subsection{Resultados}

En el anexo se detallan los resultados referidos a correlaciones entre variables. Por limitaciones de extensión, no es posible presentar resultados de cada una de las variables medidas.

\section{6.- Análisis de los datos}

Los resultados de las correlaciones de la importancia dada a las variables de RSE y su valoración, se encuentran en el apéndice. Tal como se anticipó, por motivos de extensión del presente trabajo no fue posible presentar otros resultados.

El primer elemento a destacar consiste en las elevadas calificaciones que recibe la cooperativa a lo largo de sus diferentes agencias, aún a pesar de seguir sufriendo los coletazos de la crisis del 2007-2008, la cual comenzó siendo financiera. Se esperaba que todavía tuviera una repercusión negativa en la percepción de los asociados.

En su conjunto, los atributos de la dimensión ambiental son los que presentan mayor brecha entre importancia y valoración. Es importante señalar al respecto que CoopeAnde №1 R.L ha sido la pri- 
mera cooperativa financiera certificada Carbono Neutral en Costa Rica. Sin embargo, ello fue anunciado un mes después de finalizado el trabajo de campo. Por lo tanto, a los esfuerzos por seguir progresando en materia ambiental se aúnan los referidos a comunicar a los grupos de interés los logros ya alcanzados. Por su parte, dentro de la dimensión social, "Patrocinar actividades de salud pública", "Mejorar la calidad de vida de las comunidades en las que opera" $y$ "Contar con iniciativas para mejorar el acceso a los servicios financieros a personas desfavorecidas" presentan las mayores distancias. Y, en la dimensión económica, "Contar con políticas e instrumento para la lucha contra la corrupción".

Los asociados perciben que es congruente la realización de acciones de RSE con la comercialización de productos y servicios financieros de CoopeAnde $\mathrm{N}^{\circ} 1 \mathrm{R}$.L. También muestran una positiva percepción en torno a la motivación de tales acciones. Al respecto, puede señalarse que las medias alcanzadas a nivel total son inferiores al resto de la batería de temas tratados. Sin embargo, las valoraciones no descienden de los 5.9 puntos. Asimismo, las calificaciones referidas a credibilidad son nuevamente elevadas, cercanas a los 6.5 puntos tanto en lo referido a ser competente como en cuanto a su experiencia, honestidad y compromiso con sus $\mathrm{Gl}$. Conjuntamente, se trata de una organización con elevada reputación, de acuerdo con sus asociados, con valoraciones que rondan los 6.7 puntos. En otro orden, los asociados también se declaran altamente satisfechos y con perspectivas de relaciones a largo plazo.

En cuanto a la calidad del servicio percibido, puede ser diferenciado en tres apartados: (1) calidad del servicio funcional, que se refiere a la agencia, su aspecto y diseño; (2) calidad del servicio técnico, que se vincula a los productos y sus características; y (3) calidad del servicio, centrado en los trabajadores. En términos generales, cada uno de ellos recibe calificaciones superiores a los 6.5 puntos. La calidad del servicio técnico es el apartado con valoraciones relativamente inferiores, mientras que el rol del trabajador resulta clave en el servicio prestado y en la calidad percibida. Vinculado con los resultados de Importancia y Valoración a los que se hizo referencia previamente, precisamente los ítems vinculados a trabajadores tienen las medias más elevadas en ambas variables.

En términos generales, se observa que la agencia de San José tendencialmente presenta resultados inferiores a la media, mientras que agencias como las de Guápiles, Puntarenas y Pérez Zeledón tienden a ubicarse por encima del total.

Merece la pena destacar el escaso conocimiento espontáneo relativo a proyectos o iniciativas sociales desarrolladas por la cooperativa, situación que en agencias como la de Cartago y San José representa al $36 \%$ y $31 \%$ de los encuestados, respectivamente. Dada la base asociativa, entre quienes declaran conocer ejemplos de tales iniciativas, prevalecen las menciones referidas a proyectos educativos.

Entre el tramo más joven, es decir hasta 35 años, las valoraciones son mayoritariamente inferiores, siendo estas diferencias estadísticamente significativas. Por el contrario, los resultados que representan a los mayores a 61 años, son superiores al total. 


\subsection{Relación entre la importancia de la RSE y la valoración de las acciones de RSE}

Además de los resultados obtenidos tras el análisis de los datos descriptivos de la muestra y de las valoraciones otorgadas a cada una de las variables, el estudio también trata de profundizar en la relación existente entre la importancia que los asociados le conceden a la RSE y la valoración que realizan sobre las acciones de RSE que lleva a cabo CoopAnde. Para ello, se ha llevado a cabo un análisis de fiabilidad de las escalas utilizadas para la medición de ambas variables superando éstas el valor mínimo de 0,8 del coeficiente de Cronbach. Por otro lado, para la medición de los coeficientes de correlación entre las dos variables objeto de estudio, debido a la inexistencia de normalidad en los datos se han utilizado técnicas de estudio no paramétricas: Tau_b de Kendall y Rho de Spearman.

Los datos que se muestran en las tablas 13 y 14 recogidas en los anexos, muestran una correlación positiva y significativa (a un nivel de significatividad inferior de 0,01 ) entre la variable importancia de la RSE y la variable valoración de la RSE. No obstante, cabe señalar que dicha correlación es muy baja, ya que, la correlación entre ambas variables presenta valores entre 0,2 y 0,4.

La relación positiva entre ambas variables, aun siendo escasa, puede indicar que aquellos asociados que presentan un mayor interés por las acciones de RSE de la cooperativa, valoran en mayor medida las acciones que lleva a cabo la misma y, al contrario, podrían penalizar con mayor intensidad las acciones que impacten de forma negativa en el entorno socioeconómico y medioambiental de la cooperativa.

\section{7.-Conclusiones}

CoopeAnde No1 R.L resulta ser altamente valorada y con buena imagen. Entre los asociados, los aspectos sociales son los que presentan mayor valoración. Al respecto, se observa que el carácter local y la cercanía con el entorno es un aspecto sensible, que al mismo tiempo se recubre de mayores expectativas y demandas. Por lo tanto, a la luz de los resultados presentados, cada agencia ha de desarrollar su propio plan de acción acorde a la estrategia de la Cooperativa.

Se ha podido confirmar que los aspectos vinculados a la RSE resultan importantes para los asociados, lo cual se presenta como un estímulo para la organización en lo referido a continuar por esta senda buscando espacios de mejora y fortalecimiento, así como en el ordenamiento de la diversidad de emprendimientos que se han venido desarrollando. En este sentido, los resultados parecen reflejar la necesidad de robustecer y reconsiderar el plano de la comunicación. Es decir, son múltiples 
las acciones que se han emprendido desde las diferentes agencias, incidiendo en diferentes Gl. Sin embargo, como se ha detallado en el análisis de los datos, 5 de cada 10 asociados declaró no conocer proyectos sociales de la Cooperativa. Ello, a pesar de haber constatado que los atributos de esta dimensión son los más valorados. A pesar de que la RSE no se limita a la dimensión social, estos resultados dan indicios del desconocimiento de las actividades que se han estado impulsando, más allá del núcleo del negocio de CoopeAnde №1 R.L. Por ello, la consideración de la RSE desde un plano estratégico ha de contribuir a un ordenamiento de prioridades que sean acordes a las necesidades y expectativas de los Gl de la Cooperativa.

\section{8.- Limitaciones y líneas futuras}

En cuanto a las limitaciones del presente estudio, se debe indicar que las muestras recolectadas en las agencias no permiten un análisis pormenorizado por variables demográficas para cada una de ellas. Sin embargo, se considera que por tratarse de una primera aproximación a los objetivos aquí analizados, se ha asegurado una base de análisis representativa de la población objeto de estudio. Además, se podría haber complementado el estudio cuantitativo con herramientas cualitativas, con la intención de profundizar en las percepciones de los asociados. No obstante, las limitantes de recursos y capacidades imposibilitaron que ello ocurriera en este primer estudio.

Respecto a las líneas futuras de investigación, se espera profundizar en los resultados obtenidos por variables tales como edad y género, lo cual contribuiría en el diseño de alineamientos generales estratégicos. Como se mencionó en el apartado anterior, existen indicios de que hay diferencias por perfil. Además, se espera aplicar modelos de ecuaciones estructurales con el fin de conocer en qué medida se relacionan los diferentes apartados recogidos: valoración de la RSE, su importancia, Credibilidad, Motivación, Reputación, Satisfacción, Lealtad, Calidad del Servicio Percibido. 


\section{Bibliografía}

AECA - ASOCIACIÓN ESPAÑOLA DE CONTABILIDAD Y ADMINISTRACIÓN DE EMPRESAS (2003): Marco conceptual de la responsabilidad social corporativa, AECA, Madrid.

ALAMINOS CHICA, A. \& CASTEJÓN COSTA, J. (2006): Elaboración, análisis e interpretación de encuestas, cuestionarios y escalas de opinión, Alicante: Editorial Marfil.

ARANZADI, D. (1999): "La empresa cooperativa y sus ventajas competitivas", Boletín de estudios económicos, 54, 16-23.

ARNAEZ, N., ARZADUN, P. \& GÓMEZ PESCADOR, I. (2012): MONDRAGON: integrando la RSE en su Modelo de Gestión Corporativo, VII Congreso Internacional Rulescoop, Valencia - Castellón.

ARZADUN, P. (2011): "Globalización económica y cooperativismo. Estudio empírico sobre el sector cooperativo argentino", CIRIEC-España, Revista de Economía Pública, Social y Cooperativa, 72, 215-235.

ARZADUN, P. (2012a): Estudio empírico sobre globalización del proceso productivo establecido de MONDRAGON y su impacto socioeconómico. Confluencia entre globalización, RSE, empresa y desarrollo, tesis doctoral, Universidad de Mondragón, Oñati, España.

ARZADUN, P. (2012b): "Mondragon: globalización del proceso productivo e impacto socioeconómico", Eusko Ikaskuntza, 15, 31-57.

ARZBACH, M. \& DURÁN, Á. (2011): "Protección de Depósitos para Bancos y Cooperativas de Ahorro y Crédito en América Latina", Documentos de la DGRV No.5, São Paulo, octubre.

ARZBACH, M. \& DURÁN, Á. (2010): "Regulación y Supervisión de Cooperativas de Ahorro y Crédito en América Latina y el Caribe", Documentos de la DGRV No. 3, São Paulo/San José, octubre.

BBCR - Banco Central de Costa Rica (2015): Informe mensual de coyuntura económica, División Económica, Junio de 2015, http://www.bccr.fi.cr/publicaciones/politica_monetaria_inflacion/Informe_mensual_de_coyuntura _economica_abril_2015.pdf, consultado el 27 de junio de 2015.

BECKER-OLSEN, K.L., CUDMORE, B.A. \& HILL, R.P. (2006): "The impact of perceived corporate social responsibility on consumer behavior", Journal of Business Research, 59, 1, 46-53.

BELHOUARI, A., BUENDÍA MARTÍNEZ, I., LAPOINTE, M. \& TREMBLAY, B. (2005): "La responsabilidad social de las empresas: ¿un nuevo valor para las cooperativas?", CIRIEC-España, Revista de Economía Pública, Social y Cooperativa, 53, 191-208. 
BIGNÉ, E., CURRÁS, R. \& SÁNCHEZ, I. (2009): "Brand credibility in cause-related marketing: The moderating role of consumer values", The Journal of Product and Brand Management, 18 (6), 437447.

COMISIÓN EUROPEA (2002): Libro Verde. Fomentar un marco europeo para la responsabilidad social de las empresas, Comisión Europea, Bruselas.

CONSEJO MONETARIO CENTROAMERICANO (2015): Informe Riesgo País. I trimestre de 2015, http://www.secmca.org/INFORMES/07\%20RiesgoPais/RiesgoPais.pdf, consultado el 26 de junio de 2015.

CURRÁS, R. (2007): Comunicación de la responsabilidad social corporativa: Imagen e identificación con la empresa como antecedentes del comportamiento del consumidor, tesis doctoral, Universidad de Valencia.

DEAN, D.H. (2002): "Associating the cooperation with a charitable event through sponsorship: Measuring the effects on corporate community relations", Journal of Advertising, 31 (4), 77-87.

GARCÍA DE LOS SALMONES, M., PÉREZ, A. \& RODRÍGUEZ DEL BOSQUE, I. (2010): “El proceso de lealtad hacia un servicio financiero: Análisis comparativo en función de la edad", XXII Encuentro de Profesores Universitarios de Marketing, Oviedo.

HUAYLUPO, J. (2003a): "El contexto social en el devenir de las cooperativas de ahorro y crédito. Una aproximación histórica al caso costarricense", VII encuentro de la red universitaria de las Américas "Retos y oportunidades de las cooperativas de ahorro y préstamos ante la globalización financiera", San José, Costa Rica, Octubre.

HUAYLUPO, J. (2003b): "La constitución social e histórica del cooperativismo en Costa Rica", UniRcoop, 1, 139-158.

INFOCOOP - Instituto Nacional de Fomento Cooperativo (2000): I/ Censo Nacional Cooperativo, http://www.aciamericas.coop/IMG/pdf/censo_cr.pdf, consultado el 28 de junio de 2015.

INFOCOOP - Instituto Nacional de Fomento Cooperativo (2012): III Censo Nacional Cooperativo, http://www.aciamericas.coop/lMG/pdf/censo_cr.pdf, consultado el 28 de junio de 2015.

INFOCOOP - Instituto Nacional de Fomento Cooperativo (2012): IV Censo Nacional Cooperativo, http://www.infocoop.go.cr/cooperativismo/estadistica/censo_cooperativo_2012.pdf, consultado el 28 de agosto de 2014.

ISO - International Organization for Standarization (2010): ISO 26000: Guía sobre Responsabilidad Social, ISO, Ginebra.

IZQUIERDO ALBERT, C. (2007): "La globalización neoliberal. Tendencias fundamentales. Impacto en el cooperativismo". En: Radrigán Rubio, M. (Coord.), El rol de las cooperativas en un mundo globalizado, IRECUS, Québec, 40-66. 
LAFFERTY, B.A., GOLDSMITH, R.E. \& NEWELL, S.J. (2002): "The dual credibility model: The influence of corporate and endorser credibility on attitudes and purchase intentions", Journal of Marketing Theory and Practice, 10 (3), 1-12.

Ley № 7391. Asamblea Legislativa de Costa Rica, 24 de mayo de 1994.

MASíS, P. (2009): Propuesta de implementación de un modelo organizativo orientado al cliente y basado en las personas, aplicando la herramienta Izarra 32, en una Cooperativa de Ahorro y Crédito de Costa Rica, tesis de maestría, Universidad de Mondragón, Oñati, España.

MTAS - Ministerio de Trabajo y Asuntos Sociales (2005): Informe del Foro de Expertos en RSE, MTAS, Madrid.

MUGARRA, A. (2003): "Responsabilidad social, balance social y empresa social". En: Salinas Ramos, D. (Coord.), Responsabilidad Social de las Empresas y Balance Social, Ávila: Universidad Católica de Ávila, 137-150.

OLIVER, R.L. (1980): "Conceptualization and measurement of disconfirmation perceptions in the prediction of consumer satisfaction". En: Hunt, H.K. \& Day, R.L. (eds.), Refining concepts and measures of consumer satisfaction and complaining behaviour, Bloomington: Indiana University School of Business.

OLIVER, R.L. (1997): Satisfaction: A behavioural perspective on the consumer, New York, NY: McGraw-Hill.

OIT - Organización Internacional del Trabajo (2012): Visión panorámica del sector cooperativo en Costa Rica. Una larga historia del sector, La Paz, Bolivia.

PÉREZ, A. (2011): Estudio de la imagen de responsabilidad social corporativa: formación e integración en el comportamiento del usuario de servicios financieros, tesis doctoral, Universidad de Cantabria, Santander, España.

PÉREZ, A., GARCÍA DE LOS SALMONES, M. \& RODRÍGUEZ DEL BOSQUE, I. (2009): "El efecto de las asociaciones corporativas en el componente actitudinal de la lealtad", XIX Congreso Nacional de ACEDE, Toledo.

PRADO, A., FLORES, J., PRATT, L. \& LEGUIZAMÓN, F. (2004): Manual de indicadores de Responsabilidad Social Empresarial para Costa Rica, San José: INCAE.

SERVQUAL (1988): Manual de indicadores de Responsabilidad Social Empresarial para Costa Rica, San José: INCAE.

Páginas de internet consultadas:

Banco Mundial: http://www.bancomundial.org/

CoopeAnde: http://www.coopeande1.com/

INEC - Instituto Nacional de Estadísticas y Censos de Costa Rica: http://www.inec.go.cr/

Superintendencia General de Entidades Financieras - SUGEF: http://www.sugef.fi.cr/ 


\section{Anexo}

CORRELACIONES ENTRE VARIABLES

\section{Tabla 2. Correlaciones variable Importancia de la RSE y variable valoración de la RSE}

\begin{tabular}{|c|c|c|c|c|}
\hline & & & RSEl & RSEV \\
\hline Tau_b de Kendall & RSEI & $\begin{array}{c}\text { Coeficiente de correlación } \\
\text { Sig. (bilateral) } \\
\text { N } \\
\text { Coeficiente de correlación } \\
\text { Sig. (bilateral) } \\
\text { N }\end{array}$ & $\begin{array}{c}1,000 \\
2223 \\
199 * * \\
, 000 \\
2223 \\
\end{array}$ & $\begin{array}{c}1,199^{\star \star} \\
, 000 \\
2223 \\
1,000 \\
. \\
2223\end{array}$ \\
\hline Rho de Spearman & RSEI & $\begin{array}{c}\text { Coeficiente de correlación } \\
\text { Sig. (bilateral) } \\
\text { N } \\
\text { Coeficiente de correlación } \\
\text { Sig. (bilateral) } \\
\text { N }\end{array}$ & $\begin{array}{l}1,000 \\
2223 \\
, 263^{* *} \\
, 000 \\
2223\end{array}$ & $\begin{array}{c}, 263^{\star *} \\
, 000 \\
2223 \\
1,000 \\
\cdot \\
2223\end{array}$ \\
\hline
\end{tabular}




\section{Tabla 3. Correlaciones variable Importancia de la RSE y} variable valoración de la RSE

\begin{tabular}{|c|c|c|c|c|c|c|c|}
\hline \multicolumn{2}{|c|}{ Tau_b de Kendall } & \multicolumn{2}{|c|}{ SOCI_MEDIA ECOI_MEDIA } & \multicolumn{2}{|c|}{ MEDI_MEDIA SOC_media } & \multirow{2}{*}{$\frac{\text { MED_media }}{, 207^{* *}}$} & \multirow{2}{*}{$\frac{\text { ECO_media }}{, 240^{* *}}$} \\
\hline \multirow[t]{3}{*}{ SOCI_MEDIA } & Coeficiente de correlación & 1,000 &, $453^{* \star}$ & $608^{* *}$ & $205^{\star *}$ & & \\
\hline & Sig. (bilateral) & . &, 000 &, 000 &, 000 &, 000 &, 000 \\
\hline & $\mathrm{N}$ & 2223 & 2223 & 2223 & 2223 & 2223 & 2223 \\
\hline \multirow[t]{3}{*}{ ECOI_MEDIA } & Coeficiente de correlación &, $453^{* *}$ & 1,000 &, $418^{* *}$ & $213^{* *}$ & $188^{* *}$ &, $247^{* *}$ \\
\hline & Sig. (bilateral) &, 000 & &, 000 &, 000 &, 000 &, 000 \\
\hline & $\mathrm{N}$ & 2223 & 2223 & 2223 & 2223 & 2223 & 2223 \\
\hline \multirow[t]{3}{*}{ MEDI_MEDIA } & Coeficiente de correlación & $608^{* *}$ &, $418^{\star *}$ & 1,000 & $208^{\star *}$ & ,222 $2^{\star *}$ &, $211^{\star \star}$ \\
\hline & Sig. (bilateral) &, 000 &, 000 & . &, 000 & ,000 &, 000 \\
\hline & $\mathrm{N}$ & 2223 & 2223 & 2223 & 2223 & 2223 & 2223 \\
\hline \multirow[t]{3}{*}{ SOC_media } & Coeficiente de correlación & $205^{\star *}$ &, $213^{* *}$ & ,208 & 1,000 &, $584^{* *}$ &, $553^{\star *}$ \\
\hline & Sig. (bilateral) & ,000 &, 000 &, 000 & . &, 000 &, 000 \\
\hline & $\mathrm{N}$ & 2223 & 2223 & 2223 & 2223 & 2223 & 2223 \\
\hline \multirow[t]{3}{*}{ MED_media } & Coeficiente de correlación & $207^{* *}$ & $188^{* *}$ & ,222 $2^{* *}$ &, $584^{* *}$ & 1,000 &, $549^{* *}$ \\
\hline & Sig. (bilateral) &, 000 &, 000 &, 000 &, 000 & . &, 000 \\
\hline & $\mathrm{N}$ & 2223 & 2223 & 2223 & 2223 & 2223 & 2223 \\
\hline \multirow[t]{3}{*}{ ECO_media } & Coeficiente de correlación &, $240^{* *}$ &, $247^{* *}$ &, $211^{* *}$ &, $553^{* *}$ &, $549^{* *}$ & 1,000 \\
\hline & Sig. (bilateral) &, 000 &, 000 &, 000 &, 000 &, 000 & . \\
\hline & $\mathrm{N}$ & 2223 & 2223 & 2223 & 2223 & 2223 & 2223 \\
\hline \multicolumn{8}{|c|}{ Rho de Spearman } \\
\hline \multirow[t]{3}{*}{ SOCI_MEDIA } & Coeficiente de correlación & 1,000 &, $524^{* *}$ &, $682^{* *}$ & $253^{* *}$ & ,260 & $295^{* *}$ \\
\hline & Sig. (bilateral) & . &, 000 &, 000 &, 000 &, 000 &, 000 \\
\hline & $\mathrm{N}$ & 2223 & 2223 & 2223 & 2223 & 2223 & 2223 \\
\hline \multirow[t]{3}{*}{ ECOI_MEDIA } & Coeficiente de correlación &, $524^{\star \star}$ & 1,000 &, $467^{\star \star}$ & $257^{\star \star}$ & $231^{\star *}$ & $295^{\star *}$ \\
\hline & Sig. (bilateral) &, 000 & . &, 000 & 000 & ,000 &, 000 \\
\hline & $\mathrm{N}$ & 2223 & 2223 & 2223 & 2223 & 2223 & 2223 \\
\hline \multirow[t]{3}{*}{ MEDI_MEDIA } & Coeficiente de correlación & $682^{\star \star}$ & $467^{\star \star}$ & 1,000 & $247^{\star \star}$ & $266^{* *}$ & $253^{* \star}$ \\
\hline & Sig. (bilateral) &, 000 &, 000 & . &, 000 &, 000 &, 000 \\
\hline & $\mathrm{N}$ & 2223 & 2223 & 2223 & 2223 & 2223 & 2223 \\
\hline \multirow[t]{3}{*}{ SOC_media } & Coeficiente de correlación & $253^{\star \star}$ & $257^{\star *}$ &, $247^{\star \star}$ & 1,000 & $689^{* *}$ & $649^{* \star}$ \\
\hline & Sig. (bilateral) &, 000 &, 000 &, 000 & . &, 000 &, 000 \\
\hline & $\mathrm{N}$ & 2223 & 2223 & 2223 & 2223 & 2223 & 2223 \\
\hline \multirow[t]{3}{*}{ MED_media } & Coeficiente de correlación & ,260 & $231^{* *}$ & ,266 &, $689^{* *}$ & 1,000 & $652^{* \star}$ \\
\hline & Sig. (bilateral) &, 000 &, 000 & ,000 &, 000 & . &, 000 \\
\hline & $\mathrm{N}$ & 2223 & 2223 & 2223 & 2223 & 2223 & 2223 \\
\hline \multirow[t]{3}{*}{ ECO_media } & Coeficiente de correlación & $295^{* *}$ & $295^{* *}$ & $253^{* *}$ & $649^{* *}$ & $652^{* *}$ & 1,000 \\
\hline & Sig. (bilateral) &, 000 &, 000 &, 000 &, 000 &, 000 & . \\
\hline & $\mathrm{N}$ & 2223 & 2223 & 2223 & 2223 & 2223 & 2223 \\
\hline
\end{tabular}

